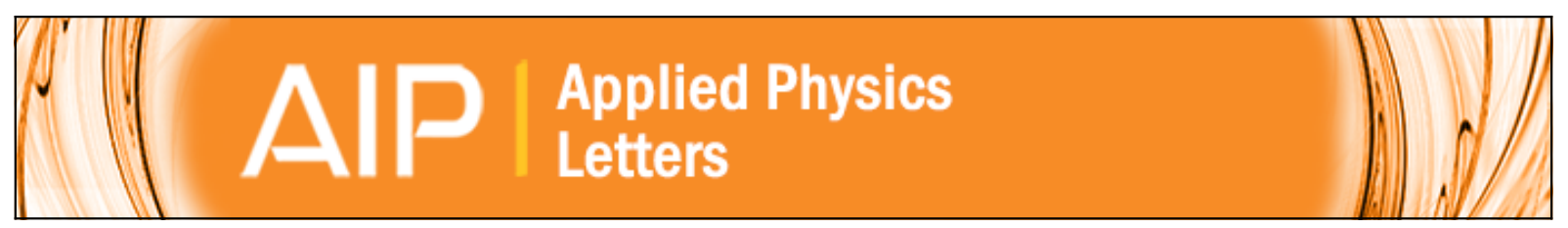

\title{
Particle in cell simulation of a radiofrequency plasma jet expanding in vacuum
}

C. Charles, R. Hawkins, and R. W. Boswell

Citation: Applied Physics Letters 106, 093502 (2015); doi: 10.1063/1.4914109

View online: http://dx.doi.org/10.1063/1.4914109

View Table of Contents: http://scitation.aip.org/content/aip/journal/apl/106/9?ver=pdfcov

Published by the AIP Publishing

\section{Articles you may be interested in}

Erratum: "Equilibrium pellet and liquid jet shape under high ablation pressures" [Phys. Plasmas5, 1380 (1998)]

Phys. Plasmas 19, 109901 (2012); 10.1063/1.4757986

Effect of helium on spatial plasma parameters in low pressure argon-helium plasma

Appl. Phys. Lett. 100, 164107 (2012); 10.1063/1.3703608

Fast electron energy deposition in a magnetized plasma: Kinetic theory and particle-in-cell simulation Phys. Plasmas 17, 033106 (2010); 10.1063/1.3356066

Particle-in-cell simulations of planar and cylindrical Langmuir probes: Floating potential and ion saturation current J. Vac. Sci. Technol. A 24, 1366 (2006); 10.1116/1.2187991

Ion orbits in a cylindrical Langmuir probe

Phys. Plasmas 13, 043501 (2006); 10.1063/1.2181971

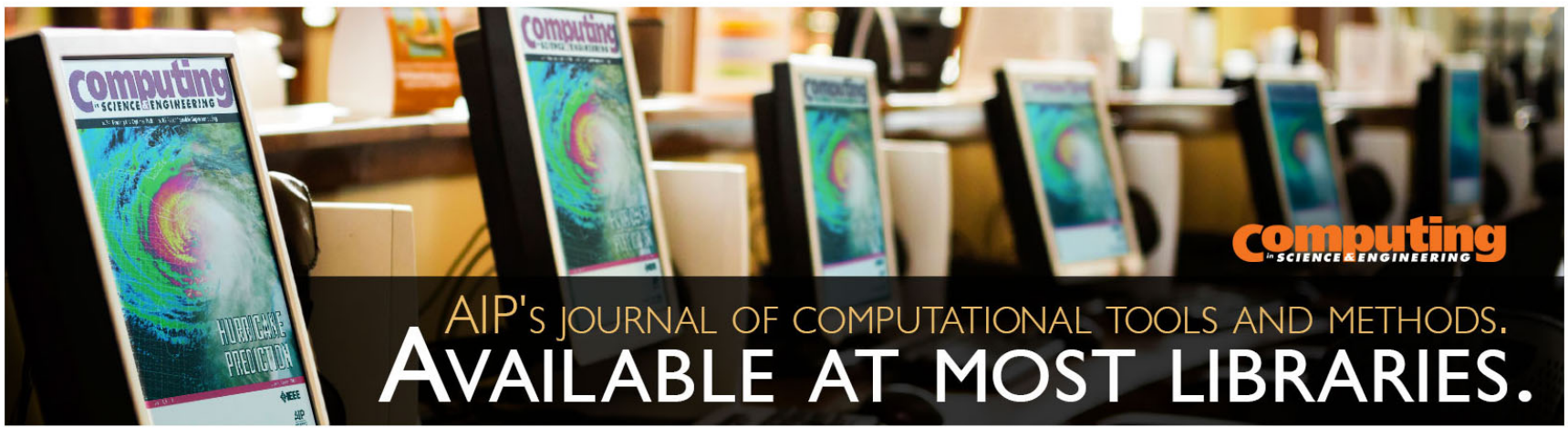




\title{
Particle in cell simulation of a radiofrequency plasma jet expanding in vacuum
}

\author{
C. Charles, ${ }^{\text {a) }}$ R. Hawkins, and R. W. Boswell \\ Space Plasma, Power and Propulsion Laboratory, Research School of Physics and Engineering, \\ The Australian National University, Canberra ACT 2601, Australia
}

(Received 15 January 2015; accepted 23 February 2015; published online 3 March 2015)

\begin{abstract}
The effect of a pressure gradient $(\sim 133 \mathrm{~Pa}-0.133 \mathrm{~Pa})$ on electron and ion energy distributions in a radiofrequency (rf at $13.56 \mathrm{MHz}$ ) argon plasma jet is studied using a 1D-3v Particle In Cell (PIC) simulation. The PIC domain is three times that of the $0.018 \mathrm{~m}$ long plasma cavity and the total simulation time is $1 \mathrm{~ms}$. Ion heating and acceleration up to a drift velocity about $2000 \mathrm{~m} \mathrm{~s}^{-1}$ are measured along the jet's main expansion axis. Elastic and charge exchange ion-neutral collisions histograms computed at equilibrium during $0.74 \mathrm{~ms}$ show that charge exchange collisions act as the main neutral heating mechanism. (C) 2015 AIP Publishing LLC.
\end{abstract}

[http://dx.doi.org/10.1063/1.4914109]

High pressure (several Torr up to atmosphere) dc (direct current) or rf (radiofrequency) plasma jets have been extensively studied ${ }^{1}$ and developed for a broad range of applications: biomedical applications and surface modifications, ${ }^{2}$ environmental protection, ${ }^{3}$ materials processing in the microelectronics industry, ${ }^{4,5}$ and hollow cathode neutralisers for space use. ${ }^{6}$ The physical properties of the jet strongly depend on the gas type and operating pressure and on its geometric expansion or interaction with a boundary. Recently, there has been increasing interest in the modelling ${ }^{7}$ and development of small size "collisional" electric thrusters aimed at generating heated neutrals via electron-neutral and ionneutral collisions in rare gases (i.e., arcjets and hollow cathodes, ${ }^{6}$ rf electrothermal micro-plasma jet, ${ }^{8}$ dielectric capillary jet ${ }^{9}$ ). Small diameter plasma discharges (a few mm down to a few hundreds of microns) usually operate at pressures above $133 \mathrm{~Pa}$ (1 Torr) and up to tens of thousands of $\mathrm{Pa}$ (hundreds of Torr) for plasma breakdown and steady-state and therefore exhibit a strong neutral pressure gradient along the jet axis. The fluid model approximation becomes debatable as the pressure decreases along the jet axis to yield free-jet expansion in vacuum. Here, a 1D-3v Particle In Cell (PIC) simulation is used to get some insight into the effect of this pressure gradient (from about $133 \mathrm{~Pa}$ to $0.133 \mathrm{~Pa}$ ) on electron and ion energy losses in a small diameter radiofrequency argon plasma micro-jet expanding in vacuum.

The MiniPR rf plasma jet has been described previously ${ }^{10}$ and consists of a $18 \mathrm{~mm}$-long, $1.5 \mathrm{~mm}$ inner diameter ceramic (alumina) plasma cavity surrounded by a central annular $5 \mathrm{~mm}$-wide rf copper electrode (fed by the $13.56 \mathrm{MHz}$ power via an impedance matching network) and two grounded $3 \mathrm{~mm}$-wide aluminium electrodes separated by two ceramic rings, $3 \mathrm{~mm}$-wide upstream (plenum side) and $4 \mathrm{~mm}$-wide downstream (vacuum chamber side) as shown in Figure 1. Here, it is mounted onto the $1 \mathrm{~m}$-long, $1 \mathrm{~m}$ diameter Irukandji vacuum chamber equipped with a turbo/ primary pumping system, an ion gauge and a convectron gauge. ${ }^{10}$ Its base pressure is about $7 \times 10^{-7}$ Torr. Argon gas

\footnotetext{
a)Email: christine.charles@anu.edu.au
}

is introduced via a $40 \mathrm{~mm}$-diam, $12 \mathrm{~mm}$-long plenum cavity (equipped with a $26 \mathrm{~mm}$-diam, $12 \mathrm{~mm}$-deep viewing window and a convectron gauge), flows through MiniPR's ceramic plasma cavity and expands into the vacuum chamber. The PIC simulation is developed so as to represent this configuration as accurately as possible, based on measured plenum and chamber pressures of $213 \mathrm{~Pa}$ (1.6 Torr) and $0.033 \mathrm{~Pa}(2.5$ $\times 10^{-4}$ Torr), respectively.

A previously reported ${ }^{11,12}$ and benchmarked ${ }^{13}$ PIC code including elastic, excitation, and ionisation electron-neutral collisions and elastic and charge exchange ion-neutral collisions in argon is rewritten from Matlab to $C^{++}$and further developed by adding a varying pressure Monte Carlo collisional routine similar to that described in Ref. 14 to be more representative of the experiment. The simulation domain is set from $x=0$ to $x=0.054 \mathrm{~m}$ with 500 grid points to be three times that of the plasma cavity length of $0.018 \mathrm{~m}$ (Fig. 1). The plenum cavity is not taken into account and the simulation is bounded by two absorbing walls at $\mathrm{x}=0$ and $\mathrm{x}=0.054 \mathrm{~m}$, respectively. Although the $\mathrm{rf}$ plasma is

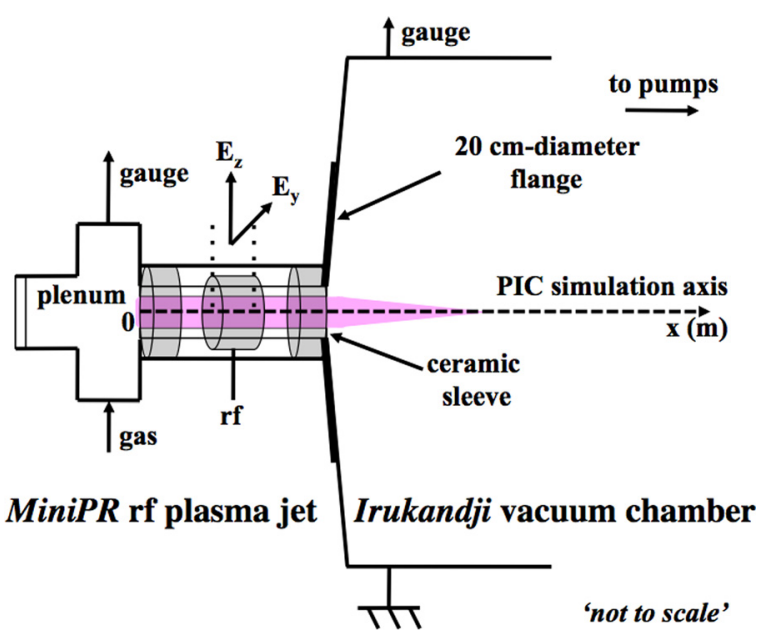

FIG. 1. Schematic of the experimental and particle in cell simulation set up showing the MiniPR radiofrequency plasma jet mounted within a vacuum flange attached to the larger Irukandji vacuum chamber. 


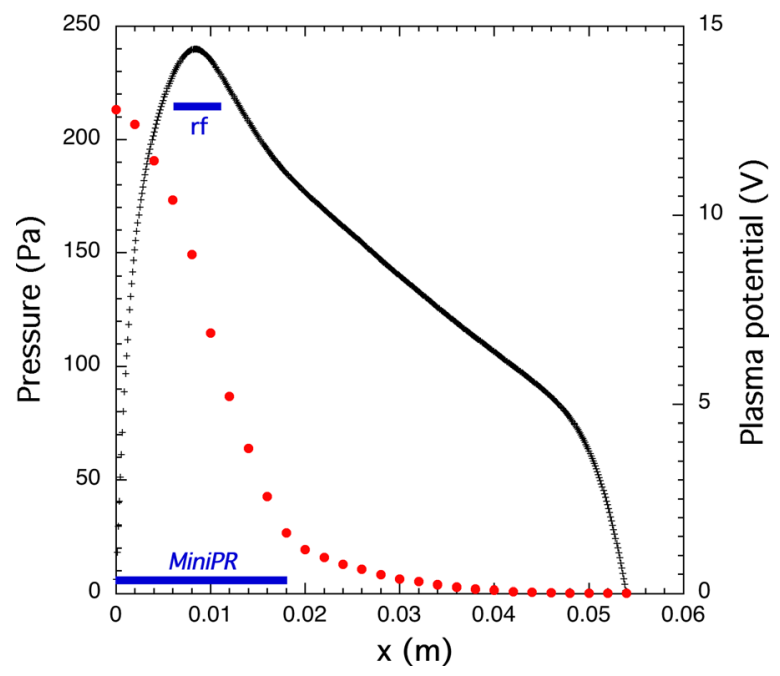

FIG. 2. Input gas pressure axial profile (red solid circles) used for the PIC simulation and based on experimental Pitot tube measurements ${ }^{15}$ and plasma potential profile (black crosses) obtained with the PIC simulation; solid horizontal blue lines show the axial position of the rf electrode and plasma cavity, respectively.

capacitively coupled, it has been shown that the maximum density resulting from operation in a Gamma mode is localised in the vicinity of the rf annular electrode $(\mathrm{x}=0.006-0.011 \mathrm{~m})$ and strongly decreases along the expansion axis. ${ }^{8,15}$ Hence, rf inductive heating at $13.56 \mathrm{MHz}$ is applied in both the $\mathrm{y}$ and $\mathrm{z}$ directions (using an input antenna current density of $5 \mathrm{~A} \mathrm{~m}^{-1}$ in each direction) from $\mathrm{x}=0.006$ to $0.011 \mathrm{~m}$ to create the generation of a localised plasma in that region ${ }^{16}$ (perpendicular rf electric field $\mathrm{E}_{y}$ and $\mathrm{E}_{z}$ shown in Fig. 1). The gas pressure profile used for the PIC simulation is shown by filled circles in Figure 2 and is based on pressure measurements made using a Pitot tube along the central axis of a $4 \mathrm{~mm}$-diam system with a similar upstream/ downstream pressure ratio of over $200 .{ }^{15}$ This work showed that the pressure in the plasma creation region was approximately halfway between the two values at the upstream (213 Pa or 1.6 Torr) and downstream (25 Pa or 0.2 Torr) ends of the plasma cavity, which is about $135 \mathrm{~Pa}$ (1 Torr) here.

The pressure gradient is simulated by a "random" neutral extracted from a Maxwellian distribution at a fixed temperature $T_{\text {gas }}$ (set at $300 \mathrm{~K}$ in this study): virtual neutrals are created with given random energy from a Maxwellian to collide with electrons and ions. There are 3 random Gaussian variables used to give the $\mathrm{x}, \mathrm{y}$, and $\mathrm{z}$ velocities. To take into account the varying axial gas pressure, the probability of collisions is calculated once at the maximum pressure (left boundary) and subsequently weighted according to the pressure profile, similarly to the method described in Ref. 14. The neutrals act as an energy sink at a fixed temperature $T_{\text {gas }}$ and this is a simplification of the actual experiment. In addition, here the ion and electron fluxes remain constant along the expansion since there is neutrality and the particle loss only occurs at the two ends of the simulation set as "absorbing" walls. Super particles which represent a large number of real particles are presently used with a typical macro-particle size of $q_{\text {factor }}=1.5 \times 10^{9}$ and a total number of macro-particles between $10^{4}$ and $10^{5}$ at equilibrium. ${ }^{17}$ Here, emphasis is made on monitoring the collisional effects

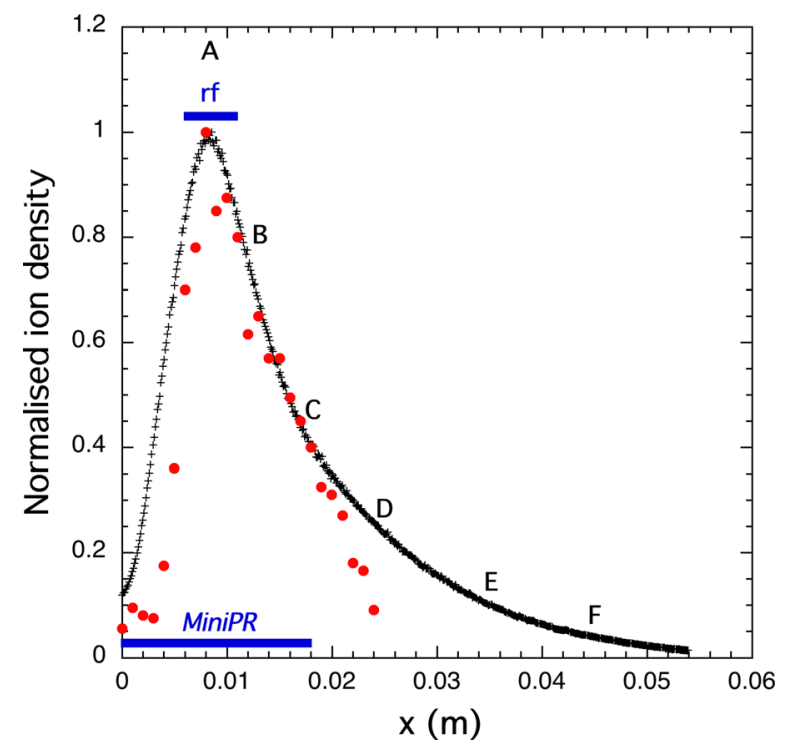

FIG. 3. Simulated (black crosses) and measured ${ }^{8}$ (red solid circles) normalised ion density axial profile; 6 axial positions labeled $\mathrm{A}-\mathrm{F}$ and corresponding to $\mathrm{x}_{A, B, C, D, E, F}=[0.0085,0.012,0.018,0.025,0.035,0.045] \mathrm{m}$ are used for the electron and ion energy distribution analysis of Figures 4 and 5.

and achieving a total simulation time of about $1 \mathrm{~ms}$, i.e., over 13 thousand $\mathrm{rf}$ cycles (using $\sim 10 \times 10^{6}$ steps with a time step $\delta \mathrm{t}=10^{-10} \mathrm{~s}$ ) and the number of macro-particles is about 22000 (a decrease in $q_{\text {factor }}$ yields a larger number of macroparticles but a much longer simulation time, without affecting the main results presented here).

Since there is no radial dimension in the PIC, only the simulated axial density normalised to 1 at its peak value is considered here and plotted along the $\mathrm{x}$ axis in Figure 3 (black crosses). The simulated density is maximum in the middle of the heating region ( $r f$ electrode) at about $\mathrm{x}=0.0085 \mathrm{~m}$ and exponentially decreases along the expansion axis. This is in agreement with a previously measured profile (solid red circles in Fig. 3) using a 4.2 mm-diam rf plasma jet prototype which allowed insertion of a movable Langmuir probe. ${ }^{8}$ Outside the plasma cavity, the simulated and measured densities are not expected to match since this experimental device of Ref. 8 had an upstream/downstream pressure gradient ratio of two. The axial plasma potential obtained with the PIC simulation is shown by crosses in Figure 2. The maximum plasma potential is found under the rf heating section (about $14.4 \mathrm{~V}$ at $\mathrm{x}=0.0085 \mathrm{~m}$ where the maximum density is also measured) and would be the result of a simple power balance and ambipolar loss of ions and electrons. The potential decreases along the expansion axis, generating an accelerating electric field for the ions. The electric field is zero at $\mathrm{x} \sim 0.0085 \mathrm{~m}$, increases to a maximum value of $\sim 0.007 \mathrm{~V} \mathrm{~m}^{-1}$ from $\mathrm{x} \sim 0.010$ to $0.014 \mathrm{~m}$ and decreases approximately linearly to a value of $0.0035 \mathrm{~V} \mathrm{~m}^{-1}$ at $\mathrm{x}=0.020 \mathrm{~m}$. This electric field value is quasi-constant from $x=0.020$ to $0.045 \mathrm{~m}$. The axial drop in potential is probably due to the pressure gradient created by the decreasing plasma density and the change in electron temperature (see below). The details of the evolution of the axial electric field are quite complicated and still being investigated. Having confirmed that the PIC simulation outputs an axial density profile which is in good agreement with the 


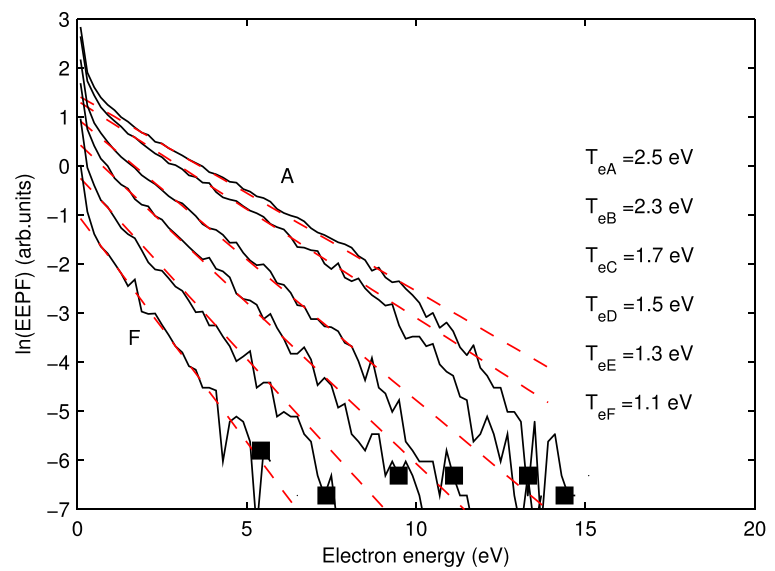

FIG. 4. Simulated EEPFs (black solid lines) obtained at positions A-F (Figure 2); the Maxwellian fits are shown in red dotted lines; the black squares track the respective plasma potential values $\mathrm{V}_{p_{A, B, C, D, F}}=$ $[14.4,13.3,11.1,9.5,7.4,5.4] \mathrm{V}$ obtained from Figure 2 (y axis positions arbitrary chosen to maintain figure clarity).

experimental findings and that the two sheaths observed at both ends of the simulation cover a small spatial extent (Figure 2), the PIC simulation is then used to investigate the particular problem of energy transfer from electrons and ions to neutrals. Applications of plasma jets to electric propulsion aim at enhancing this energy transfer, while bio-medical applications generally aim at keeping the neutrals near room temperature.

Figure 4 shows the Electron Energy Probability Function (EEPF) obtained at 6 positions along the $\mathrm{x}$ axis, respectively, corresponding to labels A-F in Figure 3: in the middle of the heating region ( $\mathrm{rf}$ electrode) at $\mathrm{x}_{A}=0.0085 \mathrm{~m}$, downstream at $\mathrm{x}_{B}=0.012 \mathrm{~m}$ (within the plasma cavity), $\mathrm{x}_{C}=0.018 \mathrm{~m}$ (at the exit of the thruster), and at $\mathrm{x}_{D, E, F}=[0.025,0.035,0.045] \mathrm{m}$ in the expansion region. The present conditions of transverse rf heating and varying axial pressure around $100 \mathrm{~Pa}$ yield non-Maxwellian EEPFs (Fig. 4). Inelastic collisions and loss of fast electrons at the axial boundaries result in a depletion at high energy as detailed by many authors. ${ }^{17-19}$ Analysis of the middle part of the EEPFs using a Maxwellian approximation leads to a maximum "bulk" electron temperature value of about $2.5 \mathrm{eV}$ at $\mathrm{x}_{A}=0.0085 \mathrm{~m}$ decreasing to $1.1 \mathrm{eV}$ at $\mathrm{x}_{F}=0.045 \mathrm{~m}$. This electron cooling process results from collisions with neutrals along the expansion. The decrease of the electron temperature along the expansion axis is correlated with the decreasing electron density and is in agreement with previous studies of thermal plasma jet expansion in low background pressure. $^{20,21}$ The value resulting from the PIC simulation near the middle of the heating region at $\mathrm{x}_{A}=0.0085 \mathrm{~m}$ is close to values measured in two experimental jets: $2.3 \mathrm{eV}$ in the heating region of a $4 \mathrm{~mm}$ diameter, $30 \mathrm{~mm}$ long plasma cavity using a rf compensated Langmuir probe, ${ }^{15} 3 \mathrm{eV}$ in the heating region of a $4.2 \mathrm{~mm}$ diameter, $20 \mathrm{~mm}$ long plasma cavity using an uncompensated probe ${ }^{8}$ and about $2 \mathrm{eV}$ using a global model and a constant cavity pressure of about $133 \mathrm{~Pa}$ (1 Torr) with neutrals at $300 \mathrm{~K}^{8}$ The values of local $^{2}$ plasma potential for positions A-F obtained from Figure 2 are shown by filled black squares in Figure 4 to compare with the respective break energy at the start of the EEPF tail

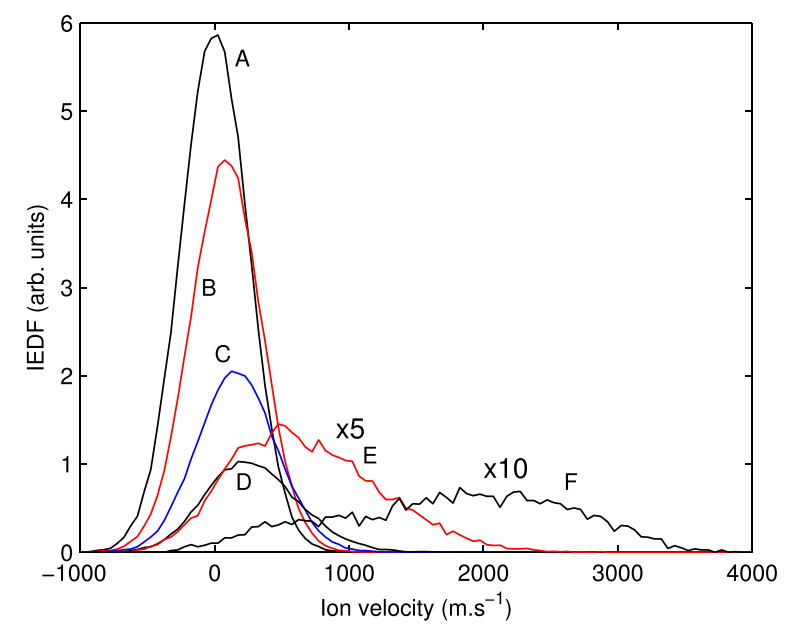

FIG. 5. Simulated IEDFs obtained at positions A-F (Figure 2); IEDFs at positions $\mathrm{E}$ and $\mathrm{F}$ are, respectively, multiplied by a factor of five and 10 for better clarity.

depletion: when the pressure is sufficiently low (positions $\mathrm{E}$ and F), this break energy is very close to the local plasma potential and reflects the electron loss to the right boundary in good agreement with simulated EEPFs in inductively coupled low pressure plasmas. ${ }^{17}$

Figure 5 shows the Ion Velocity Distribution Function (IEDF) obtained at 6 positions along the $\mathrm{x}$ axis, respectively, corresponding to labels A-F in Figure 3 (distributions E and $\mathrm{F}$ are zoomed by a factor of 5 and 10 , respectively). The IEDF widens and shifts to higher velocities along the main axis of expansion. The IEDF measured at positions $\mathrm{E}$ $(x=0.035 \mathrm{~m})$ and $\mathrm{F}(\mathrm{x}=0.045 \mathrm{~m})$, respectively, show accelerated ions characterised by an average ion drift velocity of about $600 \mathrm{~m} \mathrm{~s}^{-1}$ and $2000 \mathrm{~m} \mathrm{~s}^{-1}$ and a full width at half maximum of $1200 \mathrm{~m} \mathrm{~s}^{-1}$ and $2000 \mathrm{~m} \mathrm{~s}^{-1}$. Since the electric field is quasi constant along a large part of the expansion region, this is likely a result of the axial profile of ionization and charge exchange collisions. The latter was confirmed from the collisional histogram discussed below.

The PIC simulation is further developed to diagnose the energy transfer resulting from the ion-neutral collisions using elastic (ice) and charge exchange (icc) ion-neutral collisions histograms. Although the two histograms are cumulative and initiated at the start of the simulation, only data representative of the equilibrium state (step 2000220 to step 9399480 with a time step $\delta \mathrm{t}=10^{-10} \mathrm{~s}$ ) is used which corresponds to a total simulated time of $10000 \mathrm{rf}$ cycles $\left(\Delta t_{\text {hist }} \sim 740 \mu \mathrm{s}\right)$ and a total of about $124 \times 10^{6}$ collisions of which $60 \%$ are ice collisions. These two dimensional histograms are built using 100 axial bins from 0 to $\mathrm{L}$, the length of the system, and 200 energy bins from -7.5 to $+7.5 \mathrm{eV}$. Each ion-neutral collision is recorded as follow: the count is incremented in the (ice) or (icc) collision histogram with the axial location of the collisional event tagged to the appropriate axial bin and the corresponding energy transfer tagged to the appropriate energy bin. The energy transfer is referenced to the ion frame (since neutrals are maintained at $300 \mathrm{~K}$ ) and is calculated using $\delta E_{i}=E_{i_{\text {affer }}}-E_{i_{\text {before }}}$, where $E_{i_{\text {affer }}}$ and $E_{i_{\text {before }}}$ are the ion energy after and before the collision, respectively. If the ion looses energy to the neutral during the collision, $\delta E \leq 0$, and 
if the ion gains energy from the neutral during the collision, $\delta E \geq 0$. For each type of collision, the axial gain and loss power, respectively, correspond to (counts $\times \delta E \times q_{\text {factor }}$ $\times \mathrm{e}) / \Delta t_{\text {hist }}$, where e is the elementary charge (energy and power in the simulation are in units of $\mathrm{J} / \mathrm{m}^{2}$ and $\mathrm{W} / \mathrm{m}^{2}$ ). The three total net (gain + loss) axial powers spatially computed for (ice), (icc), and (icc + ice) collisions are found to be negative along the entire length of the system, showing that there is a net energy transfer from the ions to the neutrals at all locations independently of the collision type, in very good agreement with quasi-instantaneous neutral heating from the plasma measured recently. ${ }^{22,23}$ Analysis over the downstream side of the $\mathrm{rf}$ antenna mid-point $(\mathrm{x}=0.0085$ to $\mathrm{x}=0.0045 \mathrm{~m}$ ) shows that the ion to neutral power transfer summed over the expansion length $(x=0.018-0.045 \mathrm{~m})$ is 1.4 times that of the power summed within the cavity from $\mathrm{x}=0.0085$ to $0.018 \mathrm{~m}$. The net power transfer is dominated by the ion-neutral charge-exchange collisions compared to the ion-neutral elastic collisions both in the plasma jet cavity (net $\frac{i c c}{i c e}$ power ratio of 1.6 for $\mathrm{x}=0.0085-0.018 \mathrm{~m}$ ) and in the jet expansion region (net $\frac{i c c}{i c e}$ power ratio of 2.4 for $\mathrm{x}=0.018-0.045 \mathrm{~m})$. In the vicinity of the $\mathrm{rf}$ antenna region, the ion-neutral mean free path is small and the accelerated ions undergo many collisions with neutrals. Since the IEDF exhibits low ion temperatures in that region (Figure 5), it is expected that a lot of energy transfer occurs between ions and neutrals in the form of a large number of collisions characterized by a small energy transfer each (at low ion energy the cross sections for elastic and charge exchange are similar in amplitude). In the jet expansion, the electric field is quasiconstant and smaller in amplitude and the decreasing pressure (and increasing mean free path) allows the ions to accelerate to thousands of $\mathrm{m} \mathrm{s}^{-1}$ between collisional events (Figure 5). Ion-neutral charge exchange collisions with a large energy transfer per collision will preferentially occur, but the number of such collisions will decrease along the axis of expansion as a result of decreasing ion density and decreasing neutral density.

The charged particle current density decreases as $\frac{1}{r^{2}}$ as measured in Ref. 15 but is constant along the axis of the $1 \mathrm{D}$ simulation. In a $4 \mathrm{~mm}$ diameter prototype, a maximum axial plasma density of about $2 \times 10^{12} \mathrm{~cm}^{-3}$ is measured for $10 \mathrm{~W}$ input rf power $^{8}$ and an ion-neutral collisional model yields a neutral temperature of about $1200 \mathrm{~K}$ in argon, in agreement with a volume averaged experimental measurement of $1100 \mathrm{~K}$ using ro-vibrational spectroscopy. ${ }^{23} \mathrm{~A}$ gas temperature of $1250 \mathrm{~K}$ at $30 \mathrm{~W}$ is measured in the $1.5 \mathrm{~mm}$ diameter prototype of Fig. 1 yielding an estimated neutral gas velocity of about $800 \mathrm{~m} \mathrm{~s}^{-1}$. Hence, the thrust gain from the plasma is expected to be two to three times that of the cold gas thrust assuming a choked flow regime with cold gas at the sound speed of $300 \mathrm{~m} \mathrm{~s}^{-1}$ at $300 \mathrm{~K}$.

A 1D-3v PIC simulation was developed to model the basic expansion (axial density, potential, EEPFs, and IEDFs) of a rf plasma jet into vacuum by including a pressure gradient. A detailed analysis of the ion-neutral energy transfer was achieved by developing a histogram of all ion-neutral collisional events for an extended time period at equilibrium. The results show that ion-neutral charge exchange collisions are the dominant energy transfer mechanism contributing to neutral heating within the jet and along its expansion in vacuum.

This research was funded by the Australian Research Council Discovery Projects DP 1096653 and DP140100571.

${ }^{1}$ S. Wang, Appl. Phys. Lett. 83, 3272 (2003).

${ }^{2}$ Z. Cao, J. L. Walsh, and M. G. Kong, Appl. Phys. Lett. 94, 021501 (2009).

${ }^{3}$ K. Becker, A. Koutsospyros, S.-M. Yin, C. Christodoulatos, N. Abramzon,

J. C. Joaquin, and G. Brelles-Mario, Plasma Phys. Controlled Fusion 47, B513 (2005).

${ }^{4}$ R. Sankaran and K. Giapis, Appl. Phys. Lett. 79, 593 (2001).

${ }^{5}$ Y.-B. Guo and F. C.-N. Hong, Appl. Phys. Lett. 82, 337 (2003).

${ }^{6}$ D. M. Goebel and I. Katz, Fundamentals of Electric Propulsion (Wiley, Hoboken, USA, 2008).

${ }^{7}$ G. Makrinich and A. Fruchtman, Appl. Phys. Lett. 95, 181504 (2009).

${ }^{8}$ C. Charles and R. Boswell, Plasma Sources Sci. Technol. 21, 022002 (2012).

${ }^{9}$ A. Dunaevsky, Y. Raitses, and N. J. Fish, Appl. Phys. Lett. 88, 251502 (2006).

${ }^{10}$ C. Charles, R. W. Boswell, and A. Bish, J. Propul. Power 30, 1117 (2014).

${ }^{11}$ T. Lafleur and R. W. Boswell, Phys. Plasmas 19, 023508 (2012).

${ }^{12}$ T. Lafleur, R. W. Boswell, and J. P. Booth, Appl. Phys. Lett. 100, 194101 (2012).

${ }^{13}$ M. M. Turner, A. Derzsi, Z. Donko, D. Eremin, S. J. Kelly, T. Lafleur, and T. Mussenbrock, Phys. Plasmas 20, 013507 (2013).

${ }^{14}$ H. J. Lee and J. P. Verboncoeur, Phys. Plasmas 8, 3077 (2001).

${ }^{15}$ S. Dixon, C. Charles, R. Boswell, W. Cox, J. Holland, and R. Gottscho, J. Phys. D: Appl. Phys. 46, 145204 (2013).

${ }^{16}$ S. D. Baalrud, T. Lafleur, R. W. Boswell, and C. Charles, Phys. Plasmas 18, 063502 (2011).

${ }^{17}$ A. Meige and R. W. Boswell, Phys. Plasmas 13, 092104 (2006).

${ }^{18}$ V. A. Godyak, R. B. Piejak, and B. M. Alexandrovich, Plasma Sources Sci. Technol. 11, 525 (2002).

${ }^{19}$ Y. Takao, N. Kusaba, K. Eriguchi, and K. Ono, J. Appl. Phys. 108, 093309 (2010).

${ }^{20}$ M. C. M. van de Senden, J. M. de Regt, and D. C. Schram, Phys. Rev. E 47, 2792 (1993).

${ }^{21}$ D. M. Zube and R. M. Myers, J. Propul. Power 9, 545-552 (1993).

${ }^{22}$ A. Greig, C. Charles, N. Paulin, and R. Boswell, Appl. Phys. Lett. 105, 054102 (2014).

${ }^{23}$ A. Greig, C. Charles, R. Hawkins, and R. Boswell, Appl. Phys. Lett. 103, 074101 (2013). 